ARTICLE

DOI: $10.1038 / \mathrm{s} 41467-018-02949-y$

OPEN

\title{
Sensory deprivation in Staphylococcus aureus
}

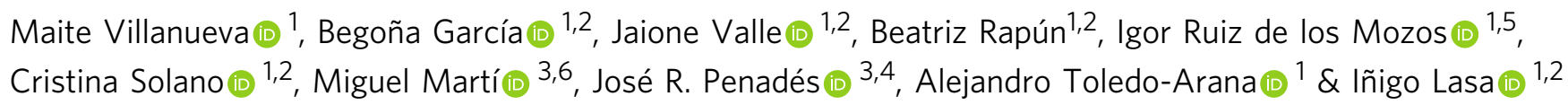

Bacteria use two-component systems (TCSs) to sense and respond to environmental changes. The core genome of the major human pathogen Staphylococcus aureus encodes 16 TCSs, one of which (WaIRK) is essential. Here we show that S. aureus can be deprived of its complete sensorial TCS network and still survive under growth arrest conditions similarly to wild-type bacteria. Under replicating conditions, however, the WalRK system is necessary and sufficient to maintain bacterial growth, indicating that sensing through TCSs is mostly dispensable for living under constant environmental conditions. Characterization of $S$. aureus derivatives containing individual TCSs reveals that each TCS appears to be autonomous and self-sufficient to sense and respond to specific environmental cues, although some level of cross-regulation between non-cognate sensor-response regulator pairs occurs in vivo. This organization, if confirmed in other bacterial species, may provide a general evolutionarily mechanism for flexible bacterial adaptation to life in new niches.

\footnotetext{
${ }^{1}$ Instituto de Agrobiotecnología. Idab, UPNA-CSIC-Gobierno de Navarra, 31006 Pamplona, Spain. ${ }^{2}$ Laboratory of Microbial Pathogenesis, Navarrabiomed, Universidad Pública de Navarra (UPNA), Complejo Hospitalario de Navarra (CHN), IdiSNA, Irunlarrea 3, 31008 Pamplona, Navarra, Spain. ${ }^{3}$ Instituto de Biomedicina de Valencia (IBV-CSIC), Jaume Roig 11, 46010 Valencia, Spain. ${ }^{4}$ Institute of Infection, Immunity and Inflammation, University of Glasgow, Glasgow G12 8TA, UK. ${ }^{5}$ Present address: Department of Molecular Neuroscience, The Francis Crick Institute, University College London Institute of Neurology, WC1B 5EH, 1 Midland Road, London NW1 1AT, UK. ${ }^{6}$ Present address: Facultad de Veterinaria y Ciencias Experimentales, Universidad Católica de Valencia San Vicente Mártir, 46001 Valencia, Spain. Maite Villanueva and Begoña García contributed equally to this work. Correspondence and requests for materials should be addressed to I.L. (email: ilasa@unavarra.es)
} 
key factor that determines the evolutionary success of a living organism is the capacity to sense environmental factors and to respond accordingly. Thus, all organisms have evolved signal transduction mechanisms to establish "functional connectiveness" between environmental cues and cellular physiology. In the case of bacteria, two-component systems (TCSs) are the primary means of the sensorial machinery ${ }^{1-3}$. A canonical two-component signaling pathway contains a histidine kinase (HK), which in response to extracellular stimuli, autophosphorylates on a conserved histidine residue. The phosphorylated $\mathrm{HK}$ then binds and transfers the phosphoryl group to a conserved aspartate residue on the response regulator (RR). The phosphorylation of the RR activates an output domain, which can then effect changes in cellular physiology often by regulating gene expression, protein interactions, or enzymatic activities. The molecular mechanisms underlying TCS signal transduction processes have been exhaustively studied since the capacity of bacterial cells to sense and respond to changes in environmental conditions is critical to understanding bacterial biology and pathogenesis, and also because TCSs are considered suitable drug targets to treat bacterial infections ${ }^{4}$.

The genome of most clinically relevant bacterial species usually encodes multiple two-component HK-RR pairs, this number being proportional to the genome size, the diversity of environments in which organisms live, and the complexity in cellular differentiation ${ }^{5,6}$. Thus, bacteria inhabiting relatively stable host environments, such as obligate intracellular parasites, possess few or even none of these signaling systems, while bacteria able to live in a variety of environments and bacteria with complex lifestyles have dozens of unique TCSs, each one potentially responding to different stimuli and thus activating a different cellular response. The number of TCSs seems to expand primarily through a mechanism of gene duplication and subsequent accumulation of mutations that insulate the new pathways from the existing twocomponent pathways ${ }^{7,8}$. The final consequence of this evolutionary process is that bacteria gain the capacity to colonize a new niche or improve the efficiency to grow under the conditions of the existing niche. In principle, if the function of a TCS remains exclusively devoted to sensing and responding to a specific environmental signal, the process of acquiring TCSs should be reversible and the successive removal of TCSs should simply reduce the number of environmental conditions under which bacteria are able to grow. This assumption has never been tested because it is presumed that the accumulation of deleterious effects caused by successive mutation of TCSs would convert free-living bacteria into uncultivable species.

Staphylococcus aureus is an important human pathogen routinely isolated as a commensal organism living in different niches, including skin, nares, and mucosal surfaces of more than a third of the human population ${ }^{9}$. From these locations, S. aureus easily leads to infections ranging from relatively mild cutaneous infections to life-threatening infections such as pneumonia, sepsis, septic arthritis, endocarditis, and osteomyelitis in people predisposed with risk factors ${ }^{10}$. The versatility of $S$. aureus as a pathogen relies on its capacity to sense, respond, and adapt the expression of a large array of virulence factors in response to the environmental cues that bacteria encounter in each tissue $e^{11-13}$. For example, $S$. aureus is able to grow at a broad range of temperatures (from 4 to $44^{\circ} \mathrm{C}$ ), oxygen levels, $\mathrm{pH}$ (from 4.5 to 8 ), salt concentrations (up to $15 \% \mathrm{w} / \mathrm{v} \mathrm{NaCl}$ ), and desiccation conditions. Taking all the above into account, we selected $S$. aureus to investigate the following questions related to the TCS network: (i) which is the minimal number of TCSs needed to sustain life in a free-living bacteria?; (ii) are TCSs self-sufficient and autonomous entities?; and (iii) does TCSs cross talk occur in vivo?
Here, using a pioneering reductionist approach by which we removed all TCS genes in two genetically independent strains, we show that $S$. aureus deprived of the TCS network is able to survive under growth arrest conditions and that a single TCS is sufficient to sustain bacterial growth. We also show that each TCS works as a self-sufficient and autonomous module able to confer adaptation to a specific niche or environmental condition and that cross-regulation between non-cognate sensor-RR pairs, though rarely, occurs in vivo.

\section{Results}

Removal of the complete TCS network from S. aureus. We initiated this study in an attempt to confirm the essentiality of TCSs for bacterial survival by removing the complete TCS network from $S$. aureus. The core genome of $S$. aureus encodes 16 TCSs (http://mistdb.com, http://www.ncbi.nlm.nih.gov/ Complete_Genomes/SignalCensus.html, http://www.p2cs.org) 6,14,15 (Fig. 1), most of which are constitutively expressed under standard laboratory conditions (Supplementary Fig. 1). We sequentially deleted the 15 non-essential TCSs, including the HKand the RR-coding genes, in two strain backgrounds, the methicillin-resistant $S$. aureus (MRSA) MW2 strain and the methicillin-sensitive $S$. aureus RN1 strain with a functional $r s b U$ gene (Fig. 2a). Note that a different deletion order was used in each strain to avoid potential deleterious combinations. Deletion of the walRK genes was not attempted at the beginning of this study because it has been shown to be essential in S. aureus ${ }^{16,17}$. The genomes of the resulting strains, named as $\Delta \mathrm{XV}$, were sequenced to identify compensatory mutations that could have arisen during the successive deletion process. We identified 10 and 5 mutations in the MW2 and RN1 derivatives, respectively. However, the absence of common mutations between both strains indicated that the deletion process did not appear to select for particular genetic changes that influenced the behavior of the mutant strains (Supplementary Table 1). Remarkably, $\Delta \mathrm{XV}$ mutant strains exhibited growth rates only slightly slower than that of the wild-type strains under aerobic conditions at $37^{\circ} \mathrm{C}$ (Fig. 2b and Supplementary Fig. 2a). These data demonstrate that S. aureus harboring a single TCS, walRK, is viable and shows a very similar growth capacity compared to wild-type bacteria when grown under standard laboratory conditions, providing experimental evidence that most TCSs are dedicated to enhance the flexibility and adaptability of bacteria to environmental signals, not being involved in the control of essential cellular processes.

Phenotypic characterization of strains deficient in TCSs. We next searched for phenotypes associated with the deletion of the TCS network in S. aureus $\triangle \mathrm{XV}$. Analysis of the metabolic capacities using API test revealed that $S$. aureus $\Delta \mathrm{XV}$ strains exhibited a deficiency only in the capacity to reduce nitrate to nitrite (Fig. 2c and Supplementary Fig. 2b). In agreement with these results, a global metabolomic profile showed that the removal of the TCS network caused significant differences in the concentration of only a few metabolites (Supplementary Data 1). These metabolites are, in general, part of the metabolism of amino acids and carbohydrates but they do not fit into any specific metabolic pathway. S. aureus $\Delta \mathrm{XV}$ also showed a growth defect at $28^{\circ} \mathrm{C}$ (Fig. 2d and Supplementary Fig. 2c); a growth defect at $\mathrm{pH} 4.5$ (Fig. 2e and Supplementary Fig. 2d); higher susceptibility to detergents (Triton X-100 gradient; Fig. $2 \mathrm{f}$ and Supplementary Fig. 2e); higher susceptibility to certain antibiotics (Supplementary Table 2); and a strong deficiency in the capacity to form abscesses in a mouse renal model ( $>1000$-fold reduction in bacterial load, $p<0.01$, Mann-Whitney test; Fig. 2g). 


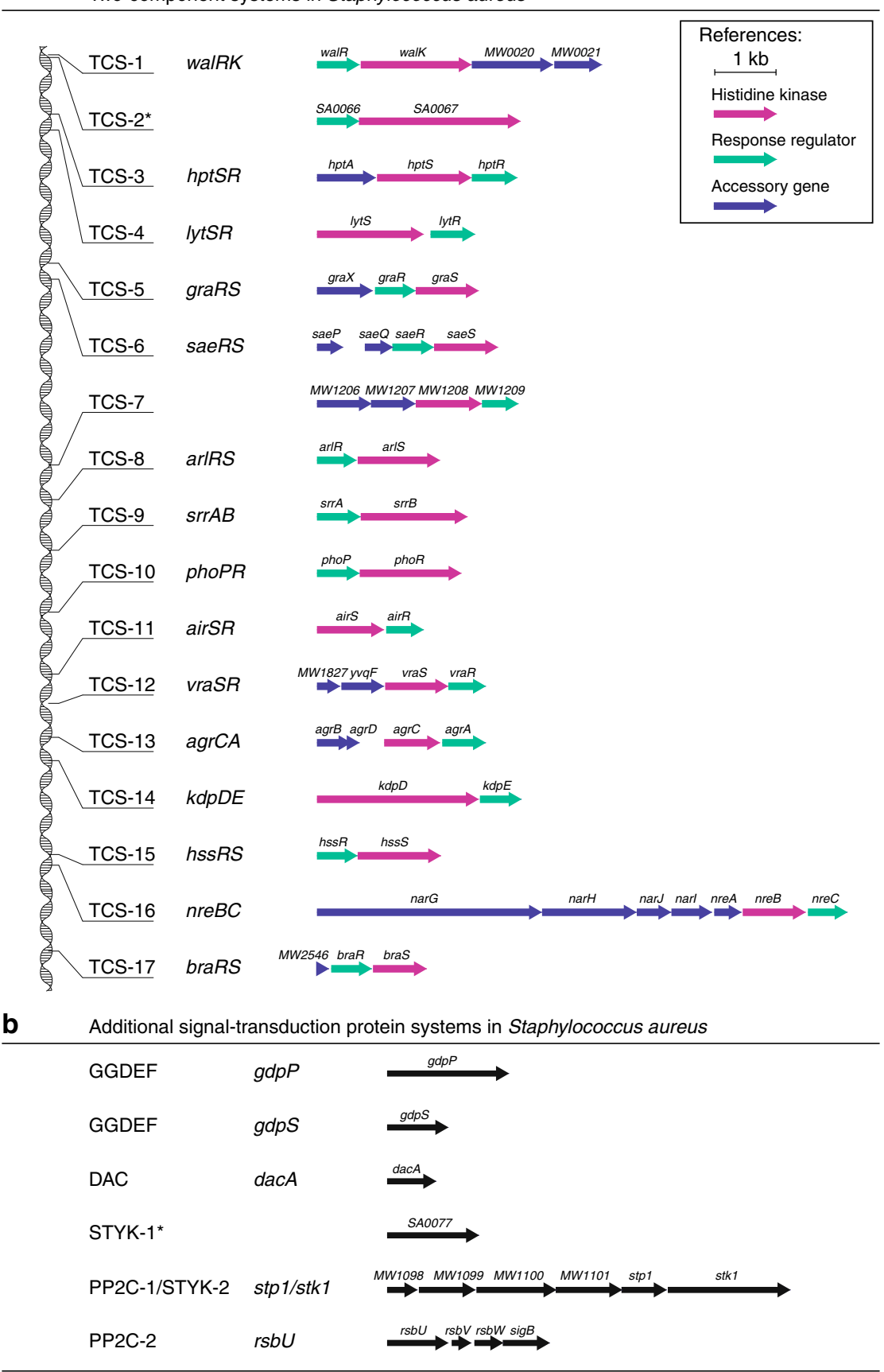

*System present in some $S$. aureus strains, which carry a particular SCCmec element.

Fig. 1 Signal transduction systems present in S. aureus. a Representation of the S. aureus genome with the 17 operons encoding two-component systems ${ }^{14}$ (http://www.ncbi.nlm.nih.gov/Complete_Genomes/SignalCensus.html). Histidine kinases are colored magenta, response regulators are colored green, and accessory genes are colored blue. Note that TCS-2 is only present in certain strains that carry a particular SCCmec element. b Additional signal transduction systems in S. aureus ${ }^{14}$ (http://www.ncbi.nlm.nih.gov/Complete_Genomes/SignalCensus.html). Genes and operons encoding the corresponding involved proteins in each signal transduction system are represented and colored black

Furthermore, $S$. aureus $\Delta \mathrm{XV}$ strain was highly attenuated since all mice infected with the MW2 mutant strain survived after 50 days of infection, while $50 \%$ of the animals inoculated with MW2 wildtype strain died by day 21 post infection (Fig. 2h). Accordingly, the expression of virulence factors (protein $\mathrm{A}$ and hemolysins) was significantly reduced in $\triangle \mathrm{XV}$ strains (Fig. $2 \mathrm{i}, \mathrm{j}$ and Supplementary Fig. $2 \mathrm{f}, \mathrm{g}$ ). Taken together, these results confirmed that TCS signaling systems allow free-living bacteria to adapt to different environmental conditions, including life in animal hosts.
TCSs are self-sufficient regulatory entities. To investigate how the complete TCS network operates as regards complementarity between different TCSs, we analyzed the aforementioned phenotypes both in a collection of single mutants in each TCS and in the collection of sequential mutants obtained during $\triangle \mathrm{XV}$ construction (Fig. 3a, b). Surprisingly, we observed that the different $\Delta \mathrm{XV}$ phenotypes appeared to be exclusively controlled by specific TCSs. Thus, the results revealed that NreBC confers the capacity to reduce nitrate to nitrite, VraSR is responsible for cell wall resistance to Triton X-100, SrrAB allows adaptation to grow at 28 
a Two-component systems

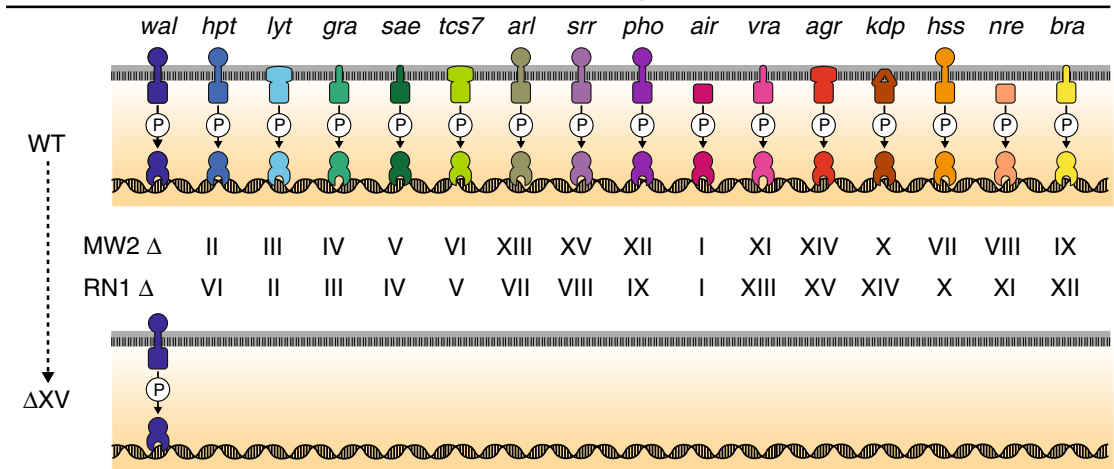

b Growth at $37^{\circ} \mathrm{C}$

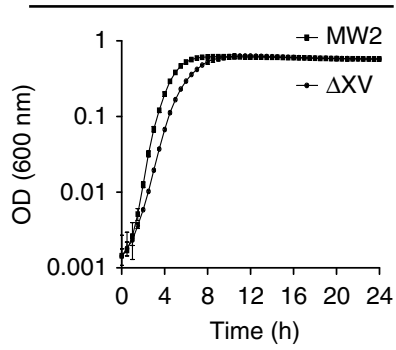

C

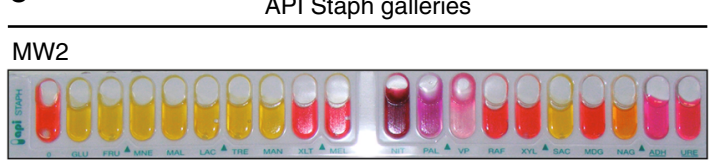

$\Delta \mathrm{XV}$

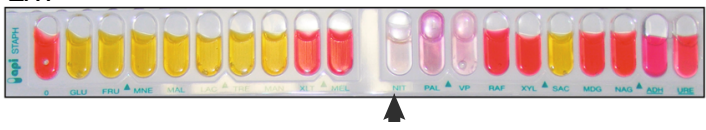

d

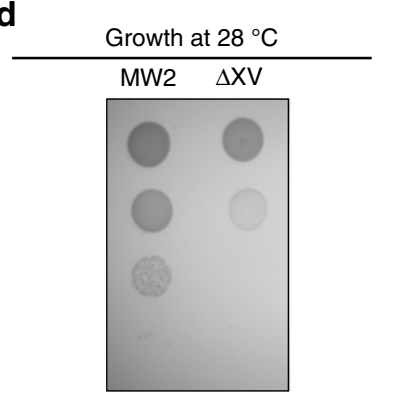

e

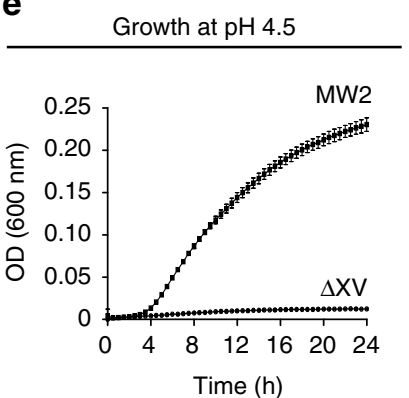

f
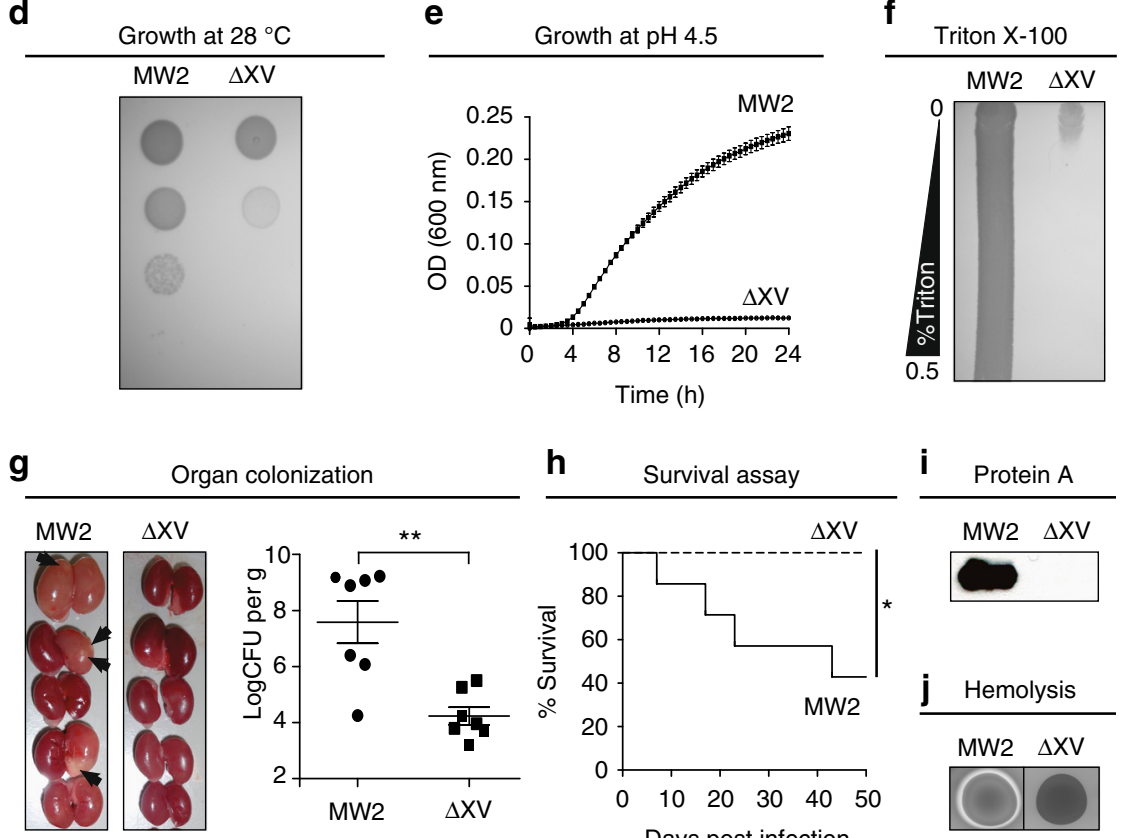

Organ colonization

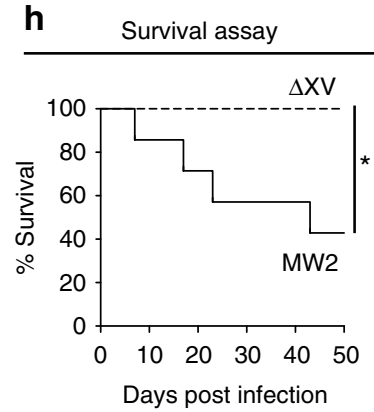

i Protein $\mathrm{A}$

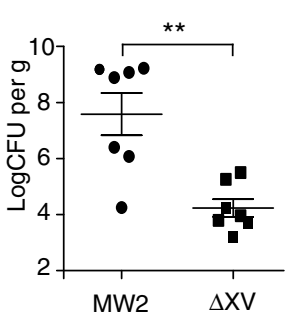

Days post infection

Fig. 2 Phenotypic characterization of a S. aureus derivative only harboring the WalRK TCS. a Scheme of the TCSs present in wild-type (MW2 and RN1) and $\triangle X V$ strains. Latin numbers of intermediate mutants sequentially constructed in order to obtain $\Delta X V$ are indicated. $\mathbf{b}$ Growth curves in TSB medium at $37^{\circ} \mathrm{C}$. Average and SD of three independent assays are represented. MW2 doubling time: 34 min; $\Delta X V$ doubling time: 42 min. c Standard metabolic pathways analyzed using commercial API Staph galleries. Only the capacity to reduce nitrate to nitrite was affected in $\Delta X V$ strain (arrowhead). d Bacterial growth on TSA medium at $28^{\circ} \mathrm{C}$. Serial dilutions were spotted on agar plates. e Growth curves in TSB medium at pH 4.5. Average and SD of three independent assays are represented. $\mathbf{f}$ Triton $\mathrm{X}-100$ sensitivity. Cultures were swabbed across agar plates containing a Triton X-100 concentration gradient. $\mathbf{g}$ Kidney colonization. Arrows indicate abscesses. Viable bacteria number per organ gram was determined. Average and SD are represented. Data were compared using the Mann-Whitney test $\left(n=7,{ }^{\star \star} p<0.01\right)$. h Survival of intravenously infected mice monitored for 7 weeks. Data were compared using a log-rank (Mantel-Cox) test $\left(n=7,{ }^{\star} p<0.05\right)$. i Protein A expression in chemically defined medium (CDM) detected by western blotting (full blot is shown in Supplementary Fig. 8). j Hemolysins production on sheep blood agar plates 
b

b

C

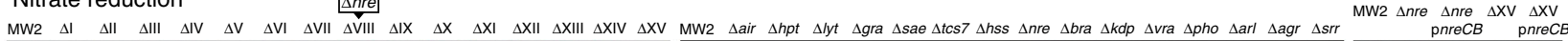

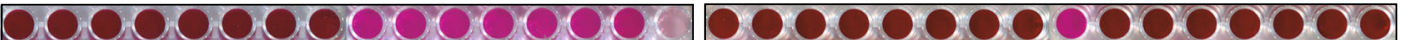
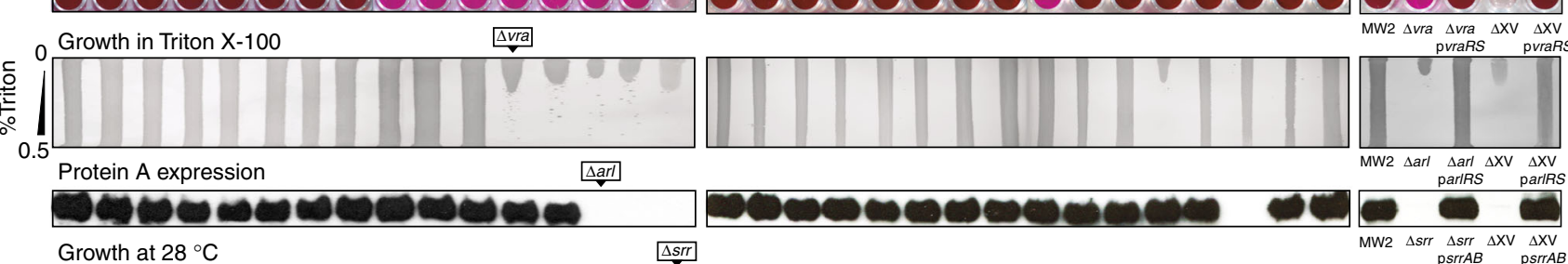

Growth at $28^{\circ} \mathrm{C}$

$\Delta s r r$
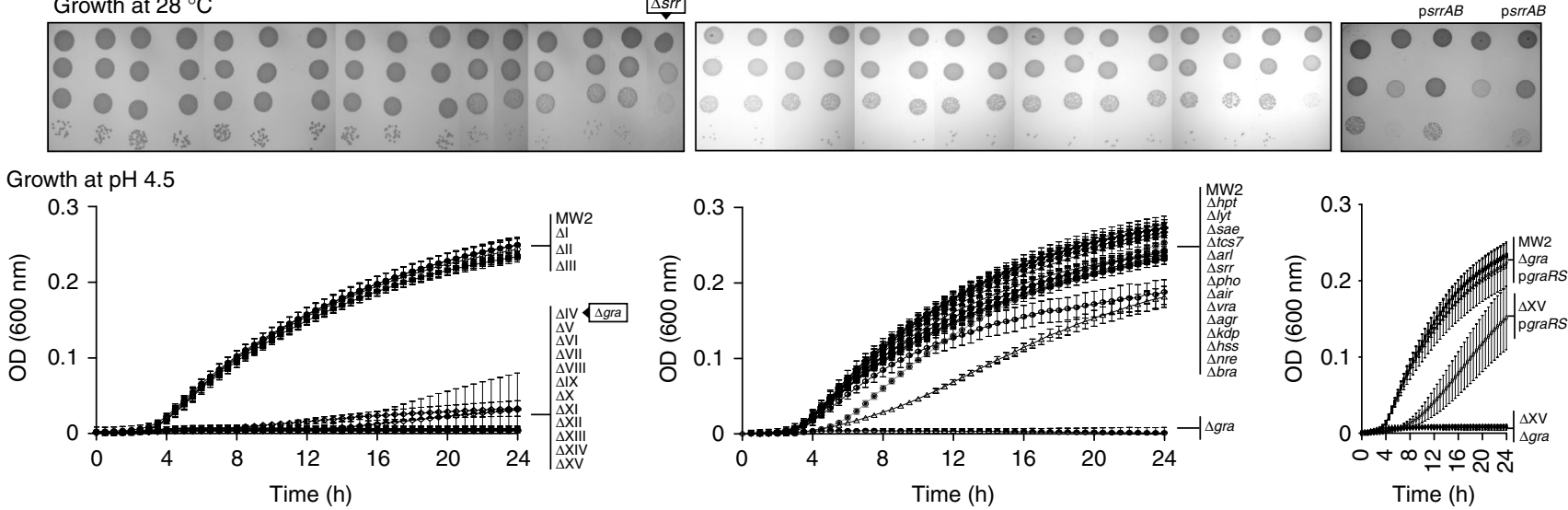

Fig. 3 Genome-wide evaluation of modularity and self-sufficiency of TCSs in S. aureus. a Phenotypes of sequential TCS mutants: (i) nitrate reduction capacity, nitrite presence was colorimetrically detected; (ii) growth capacity on Triton X-100 concentration gradient agar plates; (iii) protein A expression in CDM detected by western blotting (full blots are shown in Supplementary Fig. 9); (iv) growth ability on TSA medium at $28{ }^{\circ} \mathrm{C}$, serial dilutions were spotted on agar plates; (v) growth curves in TSB medium at pH 4.5, average and SD of three independent assays were recorded. TCSs whose mutation induces a phenotypic change are indicated. $\mathbf{b}$ Phenotypes of individual TCS mutants. Experimental conditions are described in $\mathbf{a}$. c Phenotypes of selected individual and $\triangle \mathrm{XV}$ mutant strains complemented with the corresponding TCS. Experimental conditions are described in a

${ }^{\circ} \mathrm{C}$, GraRS is necessary to grow at a low $\mathrm{pH}$, and ArlRS is necessary for transcriptional activation of protein A expression in chemically defined media $\left(\mathrm{CDM}^{18}\right.$ and RPMI). Note that protein A expression dependency on ArlRS in both chemically defined media and in three genetically unrelated $S$. aureus strains is the opposite to what it has been previously described in rich (trypticase soy broth; TSB) medium ${ }^{19}$ (Supplementary Fig. 3a and Supplementary Fig. 4a). We further explored the functional autonomy of TCSs by investigating the role of each TCS in the absence of other members of the network. For that, we made use of the $\triangle \mathrm{XV}$ genetic background, which allows the generation of derivatives, each one exclusively containing the query TCS besides WalRK. Interestingly, the ectopic expression of a copy of the corresponding TCS was sufficient to fully rescue each phenotype in $\triangle \mathrm{XV}$ strain (Figs. $3 \mathrm{c}$ and 4 ). Thus, NreCB restored the capacity to reduce nitrate to nitrite, VraRS restored the resistance to Triton $\mathrm{X}-100, \mathrm{SrrAB}$ restored the capacity to grow at $28^{\circ} \mathrm{C}$, GraRS restored growth at a low $\mathrm{pH}$, and ArlRS restored protein A expression. For the analysis of the phenotype associated to $S$. aureus $\triangle \mathrm{XV}$ virulence deficiency, ectopic complementation was not a useful strategy because the presence of the plasmid is not guaranteed in the absence of antibiotic selective pressure during the infection process. Thus, the virulence phenotype was analyzed with selected derivatives of $S$. aureus $\Delta \mathrm{XV}$ containing the agr system $(\Delta \mathrm{XV}+a g r)$ or the $s r r$ system $(\Delta \mathrm{XV}+s r r)$ restored in the chromosome. The agr system was chosen because of its major role in the production of proteases, hemolysins, and other virulence factors ${ }^{20}$, whereas the $\mathrm{sr}$ system was selected from its role in the adaptation to hypoxic and nitrosative stress encountered within the infected host ${ }^{21}$ (Supplementary Fig. 5). Evaluation of the capacity of these strains to form abscesses in a mouse renal model revealed that neither agr nor srr systems alone were capable to restore $S$. aureus virulence, despite the fact that the agr system rescued the production of hemolysins (probably $\delta$ hemolysin, since $S$. aureus MW2 is a weak producer of $\alpha$ hemolysin ${ }^{22}$ ). These results support the idea that survival in host tissues during infection very likely implies adaptation to more than one environmental condition and the production of several virulence factors, which consequently involves the participation of more than one TCS. Analysis of the dependency of specific virulence factors' expression on particular TCSs requires further investigation.

Together, these results indicate that each TCS constitutes a selfsufficient regulatory entity capable of sensing, responding, and adapting to changes in a specific environmental condition. This functional autonomy also helps to explain why the phenotypic behavior of $\triangle \mathrm{XV}$ strains is summarized as the sum of the individual phenotypes that depend on each TCS, without any synergistic cost to the bacterial fitness.

Genome-wide cross-regulation among TCSs in vivo. Studies on the specificity in the signal transduction phosphotransfer process have demonstrated that HKs typically exhibit a strong kinetic preference for their cognate $\mathrm{RRs}^{23-29}$. However, the extent to which cross-regulation occurs in vivo remains unclear, mainly because the presence of dozens of TCSs in a bacterial cell makes the analysis very complex. To answer this question, we reasoned that if ectopic expression of a native $R R$ was able to rescue a phenotype in the corresponding single TCS mutant (defective both in the $\mathrm{HK}$ and the RR pair), but not in $\Delta \mathrm{XV}$ strain, this might reflect the existence of cross talk from one or various noncognate sensor HKs present in the single mutant but absent in $\triangle \mathrm{XV}$ strain (Fig. 5a). To test this hypothesis, single mutant strains in TCSs for which a phenotype was identified (nreCB, vraRS, 
Self-suficient TCSs

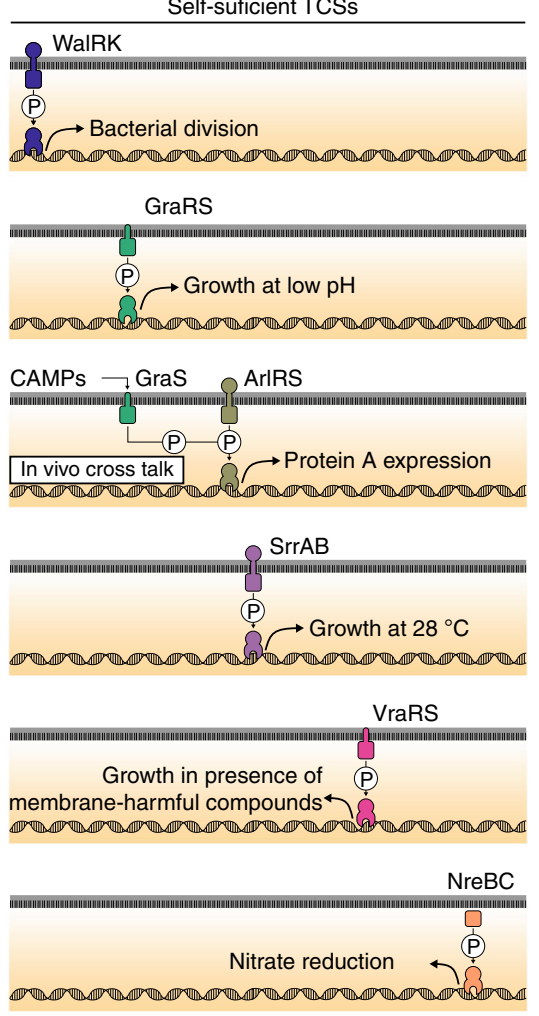

Fig. 4 Schematic summary of the S. aureus strains producing a single TCS to demonstrate functional self-sufficiency. WalRK regulates bacterial division, GraRS is necessary to grow at a low $\mathrm{pH}$, ArIRS induces the expression of protein $A$ in $C D M, \operatorname{Srr} A B$ allows adaptation to grow at $28^{\circ} \mathrm{C}$, VraRS controls the susceptibility to Triton X-100, and NreBC confers the capacity to reduce nitrate to nitrite. Moreover, GraS is able to phosphorylate ArIR, upon the presence of antimicrobial cationic peptides, such as colistin, and activates protein $\mathrm{A}$ expression

$\operatorname{arlRS}$, $\operatorname{sr} A B$, and $\operatorname{graRS}$ ), as well as the $\triangle \mathrm{XV}$ strain, were complemented with plasmids expressing the corresponding RRs. Note that the existence of a phenotype in the absence of the TCS implies that the TCS is active under the experimental conditions used in the analysis. The results revealed that plasmids expressing NreC, VraR, SrrA, and GraR were unable to restore the characteristic phenotype either in the single or in $\triangle \mathrm{XV}$ mutant strains, at least under the laboratory growth conditions tested (Fig. 5). By contrast, ArlR was able to restore protein A expression in the single $S$. aureus $\Delta$ arlRS mutant but not in $\triangle \mathrm{XV}$ mutant strain, suggesting the existence of a cross talk between ArlR and a noncognate HK (Fig. 6a). We decided to further analyze this process. We first confirmed that phosphorylation of ArlR is required to induce protein A expression. For that, the single arlRS mutant strain was complemented with a plasmid expressing the nonphosphorylable ArlR D52A protein. As shown in Fig. 6b, ArlR $\mathrm{D} 52 \mathrm{~A}$ allele was unable to activate protein A expression. Because the phosphatase activity of HKs is, in many cases, a key mechanism for eliminating the nonspecific phosphorylation of their cognate RR, we analyzed the cross-activation of ArlR expressed in a strain carrying the ArlS H242A allele. According to previous studies with EnvZ, a HK from the same family as ArlS, replacement of the conserved histidine- 242 would remove the kinase activity of ArlS preserving some level of phosphatase activity on $\mathrm{ArlR}^{30,31}$. Interestingly, the resulting strain showed a level of protein A expression similar to that observed in the single arlRS mutant strain complemented with ArlR, suggesting that the predicted phosphatase activity of ArlS $\mathrm{H} 242 \mathrm{~A}$ was unable to
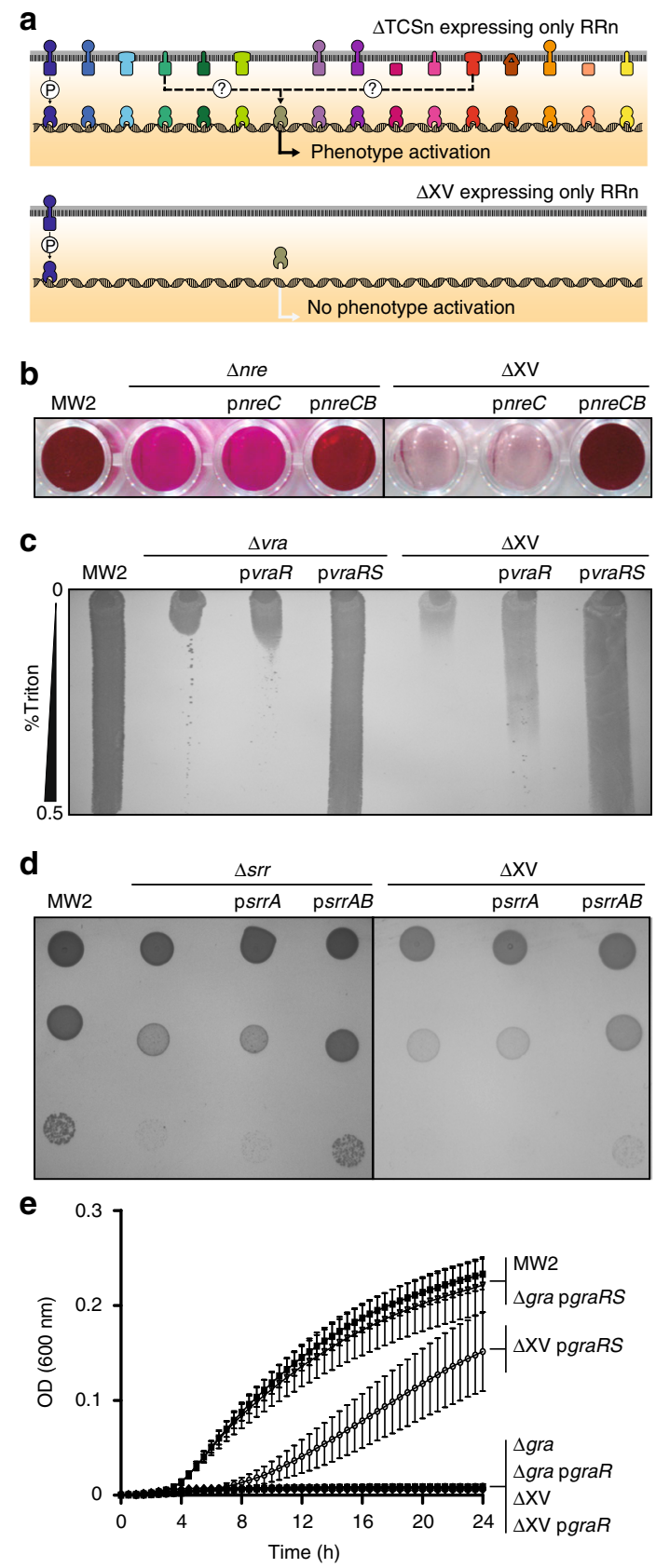

Fig. 5 Analysis of TCS cross-activation in vivo. a Scheme of the followed strategy. Ectopic expression of a RR in the corresponding single TCS mutant and in $\triangle X V$ strain. A phenotype rescue in the single TCS mutant, but not in $\Delta X V$ strain, might indicate the cross-activation from non-cognate sensor kinases. This strategy was used to analyze the cross-activation of the corresponding phenotypes. b Nitrate reduction capacity. Nitrite presence was colorimetrically detected. c Growth capacity on Triton X-100 concentration gradient agar plates. d Bacterial growth on TSA medium at $28{ }^{\circ} \mathrm{C}$. Serial dilutions were spotted on agar plates. e Growth curves in TSB medium at $\mathrm{pH}$ 4.5. Average and SD of three independent assays were recorded. Complementation with the complete TCS was used as a control in all sections

suppress cross-regulation of ArlR by other HKs (Fig. 6b). Alternatively, another explanation for these results is that mutation of the conserved histidine-242 might affect ArlS phosphatase activity.

We next sought to identify the HKs responsible for the crossphosphorylation of ArlR in vivo. To address this, a set of 15 
a

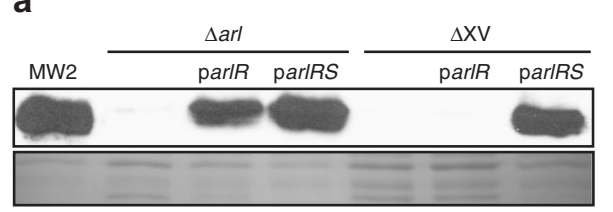

C b

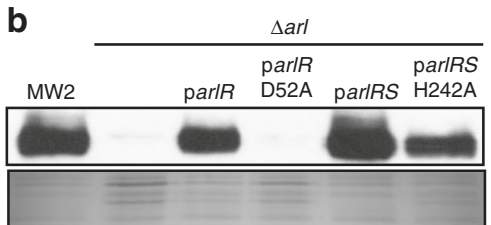

$\triangle \mathrm{XV}$ parlR

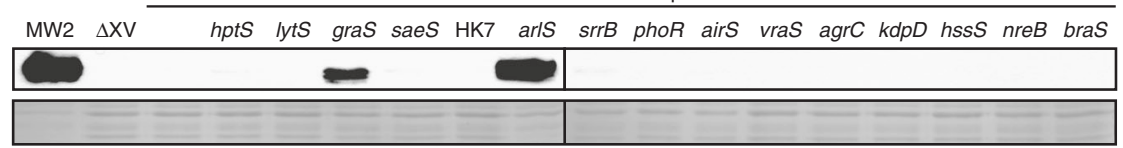

d

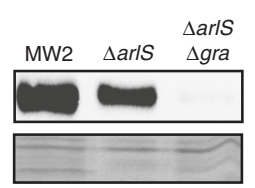

e

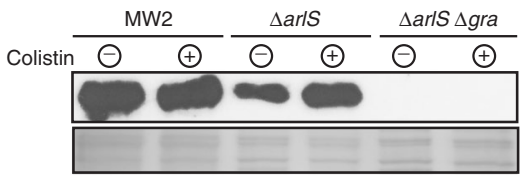

f

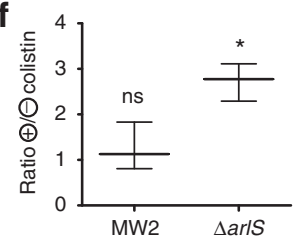

Fig. 6 In vivo cross-talk analysis. Protein A expression in CDM was detected by western blotting on the following strains (full blots and gels are shown in Supplementary Fig. 10): a $\Delta$ arl and $\Delta X V$ strains, both complemented with $\operatorname{arlR}$ and $\operatorname{arlRS} ; \mathbf{b} \Delta$ arl strain complemented with $\operatorname{arlR}$, arlR(D52A), arlRS, and arlRS (H242A); c $\Delta X V$ strain complemented with the set of plasmids containing arlR combined with the $15 \mathrm{HKs}$; d MW2, $\Delta$ arlS and $\Delta$ arlS $\Delta$ gra strains; and $\mathbf{e}$ MW2, $\Delta$ arlS and $\Delta$ arlS $\Delta$ gra strains, in the presence or absence of colistin. It is known that colistin induces the activation of GraS. Coomassie-stained gels or Ponceau-stained membranes are shown as a loading control in all sections. $\mathbf{f}$ Densitometry quantification of the protein $\mathrm{A}$ western blotting bands. Ratios of protein A band intensity from MW2 and $\Delta$ arlS strains growing in the presence or absence of colistin were represented. Average and SD of three independent assays were recorded. Data were compared using two-tailed one-sample $t$-test inferred from a hypothetical value of 1 ( $n=3$, ns $=$ no significant difference; $\left.{ }^{*} p<0.05\right)$

different plasmids, each one containing the arlR gene in combination with one non-cognate $\mathrm{HK}$, were generated and used to transform the $\Delta \mathrm{XV}$ strain (Fig. 6c and Supplementary Fig. 6). This strategy allowed us to identify GraS, in addition to ArlS used as a control of the experiment, as the HK capable to activate ArlR (Fig. 4 and Fig. 6c). As expected, activation of protein A expression disappeared in the double arlS graRS mutant (Fig. 6d, Supplementary Fig. 3b, and Supplementary Fig. 4b). Because the antibiotic colistin is able to activate $\mathrm{GraS}^{32}$, we tested whether stimulation of GraS with $50 \mu \mathrm{g} \mathrm{ml}^{-1}$ of colistin was capable of activating ArlR-dependent protein A expression (Fig. 6e, f). The results confirmed the activation of protein A synthesis in $\Delta$ arlS and not in the wild-type and $\Delta$ arlS $\Delta$ graRS strains. These data provide an example of cross-regulation that occurs at physiological protein levels and obeys to a natural input stimulus to the HK in vivo that is undetectable in the presence of the cognate ArlS. This is important because previous examples of cross-phosphorylation between a non-partner HK and RR were blind to input stimulus to the $\mathrm{HK}^{33-36}$. Interestingly, phosphorylation of ArlR by GraS does not appear to be due to a close phylogenetic relationship between them because the reciprocal phosphorylation, i.e., phosphorylation of GraR by ArlS does not detectably occur. Detailed system-level analysis with purified proteins in vitro have shown that small number of residues in the protein-protein interaction interface are responsible for the strong kinetic preference of the HK for transferring or removing the phosphate from the cognate RR pair ${ }^{8,23,25,27,37}$. Sequence identity between GraS and ArlS is $29 \%$ and $30 \%$ when the HK domain or the whole proteins are considered, respectively. Both proteins belong to the HisKA family of $\mathrm{HK}$ and they have been involved in the regulation of bacterial autolysis ${ }^{38}$, but they do not share the key residues on the $\alpha 1$ helix responsible for specificity ${ }^{27}$ (Supplementary Fig. 7). Overall, these results indicate that cross talk among different TCSs occurs in vivo.

TCSs are dispensable when bacterial growth is arrested. Intrigued by the finding that the entire TCS network, with the exception of WalRK, can be removed without affecting $S$. aureus viability, we focused our studies on the WalRK system. For this, we replaced the promoter of the walRK genes with the isopropyl$\beta$-D-thiogalactopyranoside (IPTG)-inducible Pspac promoter in $\triangle \mathrm{XV}$ strains, generating strains $\Delta \mathrm{XVI}^{\star}$ (Fig. 7a). In the absence of IPTG, the expression of walRK is turned off and the cell is deprived of the complete sensorial TCS network. The $\Delta \mathrm{XVI}^{\star}$ strains died when incubated in growth media without IPTG, confirming that walRK remains essential for bacterial viability in the absence of the remnant TCS network (Fig. 7b). For those bacteria living in multiple niches, it is very likely that they often end up in nutrient-scarce habitats. Nutrient deprivation causes growth arrest and profound physiological changes ${ }^{39}$. Thus, we sought to evaluate the necessity of WalRK TCS during starvationinduced growth arrest. Overnight cultures of $S$. aureus wild-type, $\triangle \mathrm{XV}$, and $\Delta \mathrm{XVI}^{*}$ strains (this last one grown in the presence of IPTG) were washed and resuspended in phosphate-buffered saline (PBS), dried on the surface of 24-well plastic plates, and incubated for 7 days at room temperature in the absence of IPTG. Bacterial viability was reduced to $65 \%, 55 \%$ and $55 \%$ for S. aureus wild-type, $\triangle \mathrm{XV}$, and $\Delta \mathrm{XVI}^{*}$ strains, respectively (Fig. $7 \mathrm{c}$ ). Consistent with this, depletion of the WalRK system in the wild-type strain did not affect the capacity of bacteria to survive in these conditions. From these experiments, we concluded that the TCS signal transduction network is not required for coordinating all the changes necessary to adapt bacterial physiology to starvation and desiccation. Implicit in these results is that WalRK is essential only when bacteria are dividing but dispensable when bacterial growth is arrested. The existence of a TCS essential for bacterial viability is rare and somewhat counterintuitive in that it would seem to limit the conditions under which a bacterium can thrive. It can be speculated that some TCSs might assume the control of essential processes such as cell division, DNA replication, and autolysis, because this provides direct access to the machinery involved in stopping bacterial division when environmental conditions are not adequate for growth. In this regard, Delaune et al. ${ }^{40}$ showed that overexpression of a constitutively active form 

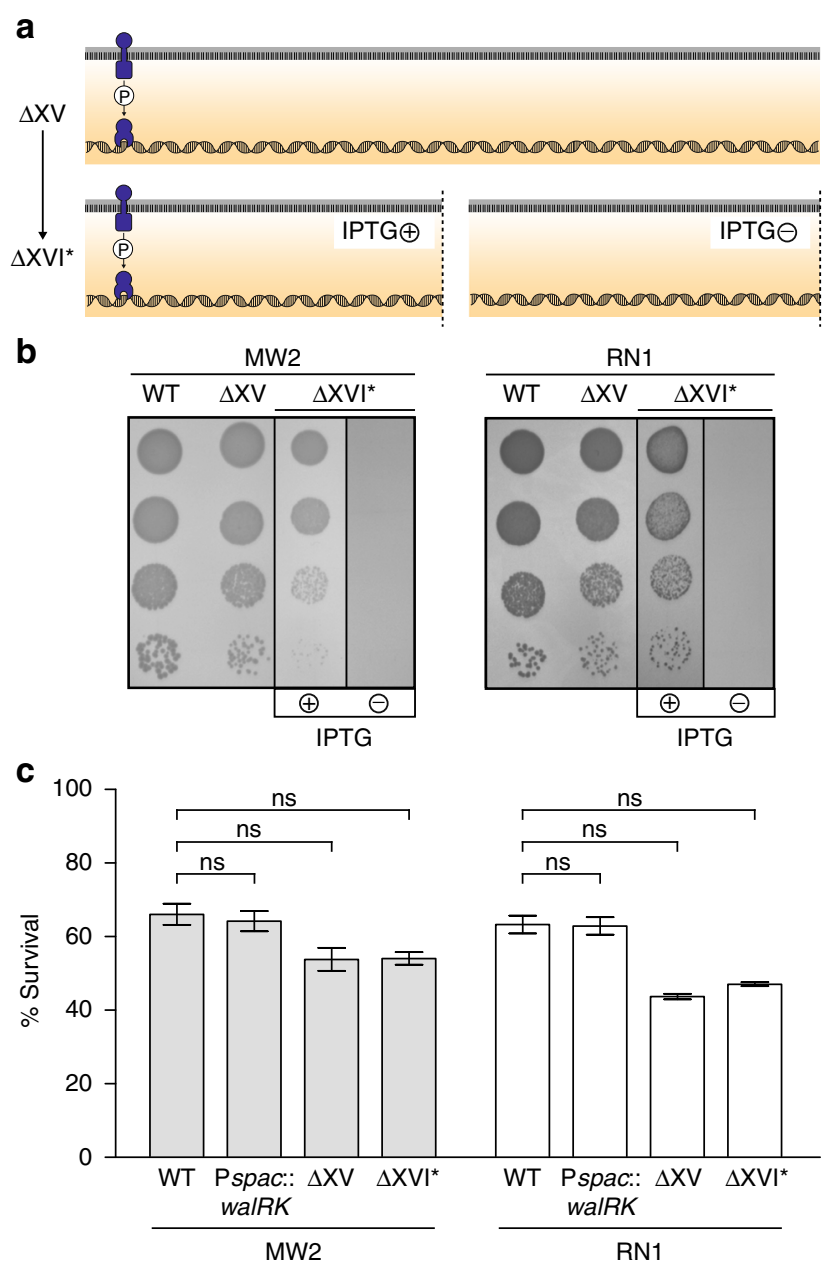

Fig. 7 Consequences of the removal of the complete TCS network in S. aureus. a Scheme of the TCSs present in $\Delta X V$ and $\Delta X V I^{\star}$ strains. $\Delta X V I^{*}$ strain harbors the walRK operon under the Pspac IPTG-inducible promoter. IPTG presence in the media controls the expression of walRK in this strain. b Growth of S. aureus strains deficient in the TCS network at $37^{\circ} \mathrm{C}$ in rich media. Drops of serial dilutions from bacterial cultures were spotted on TSA plates. IPTG was added to the media when indicated. c Survival of $S$. aureus $\Delta \mathrm{XV} I^{\star}$ during starvation-induced growth arrest. The percentage of viable cells remaining after 7 days of growth arrest was determined. Average and SD of three independent assays were recorded. Data were compared using two-tailed nonparametric Mann-Whitney test $(n=3$, ns $=$ no significant difference)

of WalR in S. aureus displayed a biphasic growth profile suggesting that constitutive WalR activation becomes detrimental once cells enter the stationary phase. Conversely, reduction of WalRK levels in the bacterial cell resulted in increased resistance to Triton X-100 and lysostaphin-induced cell lysis, and a significant decrease in peptidoglycan biosynthesis, turnover, and cell wall modifications ${ }^{41}$. It would be interesting to determine whether WalR plays a role in stopping bacterial division when environmental conditions are not appropriate for replication.

\section{Discussion}

By using a systematic deletion approach, this work provides experimental evidence supporting the idea that free-living bacteria do not need to constantly monitor environmental and intracellular parameters for survival. In agreement with the fact that some obligate intracellular bacteria lack TCS signaling, our findings indicate that free-living bacteria can grow with a single
TCS, even though growth in the laboratory still implies changes in culture parameters such as culture volume, dissolved oxygen concentration, nutrient and product concentrations, $\mathrm{pH}$, and cell density. S. aureus $\Delta \mathrm{XV}$ almost matches the definition of senseless bacterium. However, it still contains additional signal transduction systems (Fig. 1b) that include the following: (i) GdpS, the only GGDEF domain protein with a conserved GGDEF motif that is apparently unable to synthesize the secondary messenger c-di-GMP ${ }^{42,43}$; (ii) a c-di-AMP cyclase (Dac) and a phosphodiesterase (GpdP) involved in c-di-AMP metabolism ${ }^{44}$; and (iii) a Ser/Thr protein-kinase (Stk1) and two Ser/Thr proteinphosphatases (Stp1 and RsbU). Among these signal transducers, only Stk1 contains a transmembrane sensor domain that would be capable of responding to extracellular signals. Hence, though the capacity of $S$. aureus $\Delta \mathrm{XV}$ strain to sense environmental cues is certainly highly restricted, it is not null.

Taking advantage of our genetic approach, we have analyzed genome-wide the specificity of TCS signaling pathways without the interference caused by other members of the network. Our data provide an example of cross-regulation of protein A expression that occurs at physiological protein levels and obeys to a natural input stimulus in vivo that is undetectable in the presence of the cognate sensor. In this case, and since protein $\mathrm{A}$ is a moonlighting protein playing different relevant roles during $S$. aureus infection ${ }^{45}$, the most likely interpretation is that different TCSs may converge in controlling the expression of a key gene, whose expression is absolutely required for bacteria under specific and different environmental conditions. A further step will require combining the systematic analysis of cross-regulation in vivo with the spatial localization of the regulatory sensorregulator proteins.

We speculate that $S$. aureus $\Delta \mathrm{XV}$ resembles a primitive staphylococcal ancestor that, only after successive and sequentially capturing TCSs, gained the capacity to colonize new environmental niches. The set of TCSs present in S. aureus is conserved in other closely related coagulase-negative staphylococcal species such as Staphylococcus epidermidis and Staphylococcus haemolyticus. However, Staphylococcus saprophyticus, a coagulasenegative staphylococcus that is a common inhabitant of the urinary tract, contains only 11 TCSs. S. saprophyticus frequently causes uncomplicated urinary tract infections in young and middle-aged female outpatients ${ }^{46}$ without the involvement of indwelling catheters. The fact that S. saprophyticus colonizes a very narrow niche of tissues compared to $S$. aureus, suggests that the different abilities of $S$. aureus and $S$. saprophyticus to colonize different body locations and cause infection may underlay at least partially in the number of TCSs. Empirical evidence that the acquisition of additional TCSs is a dynamic process is exemplified by the presence of a new TCS in some highly pathogenic $S$. aureus MRSA strains. The new TCS is homologous to the chromosomal copy of $k d p D E$ and is carried in the SCCmec mobile element ${ }^{47}$. A definitive mechanistic insight in the process of acquisition of additional TCSs is likely to require long-term evolutionary experiments in which bacteria gain a selective advantage through the acquisition of a new TCS. We anticipate that the $S$. aureus $\triangle \mathrm{XV}$ strain could represent an excellent model organism for this purpose.

\section{Methods}

Bacterial strains, plasmids, primers, and culture conditions. Bacterial strains, plasmids, and oligonucleotides are listed in Supplementary Table 3, Supplementary Table 4, and Supplementary Table 5, respectively. Escherichia coli strains were grown in LB broth (Pronadisa). S. aureus strains were grown in TSB (Pronadisa), the chemically defined medium CDM (Hussain-Hastings-White modified medium $)^{18}$, and RPMI (Gibco Ref: 61870-010). When required for growth or selection, the medium was supplemented with IPTG, $1 \mathrm{mM}$, or/and appropriate antibiotics at 
the following concentrations: erythromycin, 1.5 and $10 \mu \mathrm{g} \mathrm{ml}^{-1}$; ampicillin, $100 \mu \mathrm{g} \mathrm{ml}^{-1}$; chloramphenicol $10 \mu \mathrm{g} \mathrm{ml}^{-1}$; and colistin, $50 \mu \mathrm{g} \mathrm{ml}^{-1}$.

DNA manipulations and bacterial transformation. Routine DNA manipulations were performed using standard procedures unless otherwise indicated. Plasmids were purified using the NucleoSpin Plasmid miniprep kit (Macherey-Nagel) according to the manufacturer's protocol. FastDigest restriction enzymes and Rapid DNA ligation kit (Thermo Scientific) were used according to the manufacturer's instructions. Plasmids were transformed into E. coli XL1-Blue strain (Stratagene) by electroporation and then introduced in S. aureus by electroporation using a previously described protocol ${ }^{48}$. Staphyloccocal electrocompetent cells were generated as previously described ${ }^{49}$. Transformation of final $S$. aureus strains with pMAD and pSD3-3 plasmids were performed by phage transduction as described previously ${ }^{50}$. Note that for transductions of plasmids to MW2 and RN1 strains, $\phi 85$ phage and $\$ 11$ phage were used, respectively.

Transcriptome analysis by tiling arrays. Complete transcriptome maps from several $S$. aureus strains obtained by tiling arrays and previously published ${ }^{51}$ (http://staph.unavarra.es/) were used to extract the transcriptional expression data from TCS operons. Images from IGB software showing the regions of the genome that encode the TCSs were extracted and shown separately (Supplementary Fig. 1).

Allelic exchange of chromosomal genes. To generate deletion mutants, we amplified by PCR two fragments of at least $500 \mathrm{bp}$ that flanked the left (primers A and B, Supplementary Table 5) and right sequences (primers C and D, Supplementary Table 5) of the region targeted for deletion. Chromosomal DNA from $S$. aureus 15981 strain was used as genomic template and Biotools DNA polymerase (Biotools) was used to carry out the PCR reactions. The PCR products (AB and CD fragments or AD fragments obtained by overlapping PCR, depending on the TCS, see Supplementary Table 5) were purified and cloned separately in the pGEM-T Easy vector (Promega). Cloned fragments were digested, purified, and fused by ligation into the shuttle vector $\mathrm{pMAD}^{52}$. pMAD ligations were electroporated into E. coli XL1-Blue (Stratagene) generating the pMAD::TCSsAD plasmid collection (Supplementary Table 4). Plasmids were purified from E. coli XL1-Blue and transformed into $S$. aureus $\mathrm{RN} 4220$ by electroporation and then introduced to MW2 and RN1 strains by phage transduction. Homologous recombination experiments were performed as described ${ }^{53}$. Erythromycin-sensitive white colonies, which did not further contain the pMAD plasmid, were tested by PCR using primers E and F (Supplementary Table 5).

The pMAD::TCS14AD(RN1) plasmid was constructed as described above, using kdp-RN1 primers (Supplementary Table 5) and RN1 chromosomal DNA as template. This plasmid was used to perform the allelic exchange of $k d p D E$ genes in RN1 strain because the flanked sequences of the region targeted for deletion differed significantly in this strain.

To restore deleted TCSs in the chromosome of $\triangle \mathrm{XV}$ strain, the TCS and the flanking sequences were amplified by PCR using primers A and D (Supplementary Table 5). Chromosomal DNA from S. aureus MW2 strain was used as genomic template and Phusion High-Fidelity DNA Polymerase (Thermo Fisher) was used to carry out the PCR reactions. The PCR products were purified and cloned in the pJET vector (Thermo Fisher Scientific). Cloned fragments were digested, purified, and fused by ligation into the shuttle vector $\mathrm{pMAD}^{52}$. pMAD ligations were electroporated into E. coli XL1-Blue (Stratagene) generating the pMAD::TCS plasmids (Supplementary Table 4). Plasmids were purified from E. coli XL1-Blue and transformed into S. aureus RN4220 by electroporation and then purified and introduced into MW2 $\triangle \mathrm{XV}$ strain by electroporation. Homologous recombination experiments were performed as described ${ }^{53}$. Erythromycin-sensitive white colonies, which did not further contain the pMAD plasmid, were tested by PCR using primers E and F (Supplementary Table 5).

To generate the insertional mutation of the walRK operon, the mutation present in RN4220 Pspac- $y y c F$ strain and generated by the insertion of the pSD3-3 plasmid ${ }^{17}$ was transferred by phage transduction to the wild-type and $\Delta \mathrm{XV}$ strains, generating Pspac::walRK and $\Delta \mathrm{XV}$ Pspac::walRK $\left(\Delta \mathrm{XVI}^{*}\right)$ strains in MW2 and RN1 strains. The resulting mutants harbored the entire walRK operon under the control of the Pspac IPTG-inducible promoter and were grown in TSA supplemented with Clo $\left(10 \mu \mathrm{g} \mathrm{ml}^{-1}\right)$. IPTG $1 \mathrm{mM}$ was added to allow the growth of these strains. Plasmid insertion was confirmed by PCR using pSD3.3.1/WalR-Fw primers (Supplementary Table 5).

\section{Identification of spontaneous mutations in $\Delta \mathbf{X V}$ strains. Identification of} genetic variations present in $\Delta \mathrm{XV}$ strains relative to the corresponding wild-type strains was carried out by computational comparison of genomic sequences generated by high-throughput sequencing. Briefly, genomic DNA was prepared from an overnight culture of parental (MW2 and RN1) and mutant (MW2 $\Delta$ XV and RN1 $\Delta X V)$ strains grown in TSB at $37^{\circ} \mathrm{C}$, as described previously ${ }^{54}$ and sequenced on an Illumina Genome Analizer IIx instrument (Genomics Platform of CIBIR, La Rioja, Spain). Reads (6M-9M 150 bp) were assembled de novo using SOAPdenovo2 algorithm ${ }^{55}$. The reads were assembled into 466 contigs ( $>100$ bp in size) corresponding to $2864441 \mathrm{bp}$ in length, with a N50 value of 16063 and a GC content of $32.67 \%$ for MW2; 236 contigs ( $>100$ bp in size) corresponding to 2856
644 bp in length, with a N50 value of 50702 and a GC content of $32.76 \%$ for MW2 $\Delta \mathrm{XV} ; 283$ contigs ( $>100 \mathrm{bp}$ in size) corresponding to $2839577 \mathrm{bp}$ in length, with a N50 value of 60685 and a GC content of $32.80 \%$ for RN1; and 292 contigs (>100 bp in size) corresponding to 2807518 bp in length, with a N50 value of 79329 and a GC content of $32.80 \%$ for RN1 $\Delta X V$. Subsequently, contigs were ordered with Mauve $^{56}$ and consensus genomic sequences containing 35 contigs for MW2, 12 contigs for MW2 $\triangle \mathrm{XV}, 17$ contigs for RN1, and 17 contigs for RN1 $\Delta \mathrm{XV}$ were manually generated using SnapGene software version 3.0.3 (GSL Biotech LLC, Chicago, USA). Finally, spontaneous and generated mutations present in $\triangle \mathrm{XV}$ strains relative to the corresponding wild-type reference sequences were analyzed using the computational pipeline breseq ${ }^{57}$. Spontaneous mutations generated during the construction of $\Delta \mathrm{XV}$ strains are presented in Supplementary Table 1.

Growth kinetics. To compare the growth kinetics of bacteria and their growth ability at a low $\mathrm{pH}$, overnight cultures grown in TSB medium at $37^{\circ} \mathrm{C}$ were diluted in TSB to an $\mathrm{OD}_{600 \mathrm{~nm}}=0.1$, and $5 \mu \mathrm{l}$ of the diluted culture were used to inoculate 96 microtiter wells (Thermo Fisher Scientific, Hemel Hempstead, UK) containing $195 \mu \mathrm{l}$ of media per well (TSB pH 7 or $\mathrm{pH} 4.5$, according to each case). Duplicates were inoculated for each strain tested. Plates were incubated at $37^{\circ} \mathrm{C}$ for $24 \mathrm{~h}$ and the growth was monitored measuring the $\mathrm{OD}_{600 \mathrm{~nm}}$ every $30 \mathrm{~min}$ using the thermostatizated spectrophotometer SpectraMAX 340PC (Molecular Devices). The average and SD of three independent assays were plotted.

Biochemical characterization. API Staph galleries (BioMérieux) were used to analyze bacterial metabolic patterns, according to the manufacturer's instructions Homogeneous bacterial suspensions with turbidity equivalent to $0.5 \mathrm{McF}$ arland were prepared using the API Staph Medium. The microtubes were then filled with the bacterial suspensions and the strip was incubated inside the incubation box at $37^{\circ} \mathrm{C}$ for $24 \mathrm{~h}$. Changes in bacterial metabolic patterns were analyzed comparing results obtained with corresponding mutants and with the wild-type $S$. aureus strain.

Quantitative metabolomics. Hydrophilic interaction liquid chromatography (HILIC) was carried out on a Dionex UltiMate 3000 RSLC system (Thermo Fisher Scientific) using a ZIC-pHILIC column $(150 \mathrm{~mm} \times 4.6 \mathrm{~mm}, 5 \mu \mathrm{m}$ column, Merck Sequant). The column was maintained at $30^{\circ} \mathrm{C}$ and samples were eluted with a linear gradient $(20 \mathrm{mM}$ ammonium carbonate in water, $\mathrm{A}$, and acetonitrile, $\mathrm{B})$ over $26 \mathrm{~min}$ at a flow rate of $0.3 \mathrm{ml} \mathrm{min}^{-1}$ as follows: $0 \mathrm{~min} 20 / 80(\% \mathrm{~A} / \% \mathrm{~B}) ; 15 \mathrm{~min} 80 /$ $20(\% \mathrm{~A} / \% \mathrm{~B}) ; 15 \mathrm{~min} 95 / 5(\% \mathrm{~A} / \% \mathrm{~B}) ; 17 \mathrm{~min} 95 / 5(\% \mathrm{~A} / \% \mathrm{~B}) ; 17 \mathrm{~min} 20 / 80(\% \mathrm{~A} / \%$ $\mathrm{B})$; and $24 \mathrm{~min} 20 / 80(\% \mathrm{~A} / \% \mathrm{~B})$. The injection volume was $10 \mu \mathrm{l}$ and samples were maintained at $5{ }^{\circ} \mathrm{C}$ prior to injection. For the mass spectrometry (MS) analysis, a Thermo Orbitrap QExactive (Thermo Fisher Scientific) was operated in polarity switching mode and the MS settings were as follows: resolution 70000 ; AGC 1e6; $\mathrm{m} / z$ range $70-1050$; sheath gas 40 ; auxiliary gas 5 ; sweep gas 1 ; probe temperature $150{ }^{\circ} \mathrm{C}$; and capillary temperature $320^{\circ} \mathrm{C}$.

For positive mode ionization: source voltage $+3.8 \mathrm{kV}$; S-Lens RF Level 30.00; SLens voltage $-25.00(\mathrm{~V})$; Skimmer voltage $-15.00(\mathrm{~V})$; Inject Flatopole Offset -8.00 $(\mathrm{V})$; and Bent Flatapole DC $-6.00(\mathrm{~V})$. For negative mode ionization: source voltage $-3.8 \mathrm{kV}$.

The calibration mass range was extended to cover small metabolites by inclusion of low-mass calibrants with the standard Thermo calmix masses (below $m / z 138$ ), butylamine (C4H11N1) for positive ion electrospray ionization mode $(\mathrm{m} / z$ 74.096426), and COF3 for negative ion electospray ionization mode $(\mathrm{m} / \mathrm{z}$ 84.9906726). To enhance calibration stability, lock-mass correction was also applied to each analytical run. Positive Mode Lock masses, number $=3$ : Lock Mass \#1 ( $\mathrm{m} / \mathrm{z})$ 83.0604; Lock Mass \#2 ( $\mathrm{m} / \mathrm{z})$ 149.0233; and Lock Mass \#3 (m/z) 445.1200 Negative Mode Lock masses, number $=1$ : Lock Mass \#1 $(\mathrm{m} / z)$ 89.0244. For data processing, instrument raw files were converted to positive and negative ionization mode mzXML files. These files were then analyzed using the XCMS/MZMatch/ IDEOM pipeline to produce the IDEOM file ${ }^{58-60}$.

Growth at $28^{\circ} \mathrm{C}$. To establish growth kinetics at $28^{\circ} \mathrm{C}$, overnight cultures were adjusted to an $\mathrm{OD}_{600 \mathrm{~nm}}$ of 1 and serially diluted in TSB. A volume of $5 \mu \mathrm{l}$ of diluted cultures were spotted onto TSA plates supplemented with the appropriate antibiotics if necessary, and plates were incubated at $28^{\circ} \mathrm{C}$ for $24 \mathrm{~h}$. Representative pictures were taken.

Triton resistance test. Triton gradient agar plates $(0-0.5 \%)$ were used to compare the resistance phenotype against Triton X-100 (USB) ${ }^{61}$. OmniTray single-well plates (NUNC) were filled with TSA $0.5 \%(\mathrm{v} / \mathrm{v})$ Triton X-100, and a slope was created when the media was still in liquid state. After media solidification, plates were totally filled with TSA, generating the triton gradient. Overnight cultures of the strains were diluted in TSB to an $\mathrm{OD}_{600 \mathrm{~nm}}=0.4$ and cell suspensions were swabbed across the agar plates containing triton concentration gradients. Plates were incubated at $37^{\circ} \mathrm{C}$ for $24 \mathrm{~h}$. Triton sensitivity was observed as a growth deficiency on the concentrated area of the plate. 
Antibiotic resistance. Antimicrobial susceptibility tests were performed at the University Clinic of Navarra. Antibiotics routinely used in clinic for the treatment of $S$. aureus infections were tested using VITEK 2 system (bioMérieux) according to the manufacturer's instructions.

Mouse infection models. Strains were cultured overnight in TSA plates at $37^{\circ} \mathrm{C}$ and a single colony was resuspended into $5 \mathrm{ml}$ of PBS to an $\mathrm{OD}_{600 \mathrm{~nm}}=0.2\left(1 \times 10^{8}\right.$ colony-forming units (CFU) per $\mathrm{ml}$ ). For the kidney colonization assays, 5-weekold CD1 female mice (Charles-River) were inoculated by eye vein injection with $100 \mu \mathrm{l}\left(1 \times 10^{7}\right.$ cells $)$ of these diluted suspensions of $S$. aureus. Groups of seven mice were used for each strain tested. After 1 week, mice were euthanized and kidneys were removed. Pictures of the kidneys were taken to show abscess formation and then the organs were homogenized in PBS $\left(9 \mathrm{ml} \mathrm{g}^{-1}\right)$, serial dilutions were performed and spread on TSA plates in triplicate for determination of the number of CFU per organ gram. For survival assays, 5-week-old CD1 female mice were inoculated by eye vein injection with $100 \mu \mathrm{l}\left(1 \times 10^{7}\right.$ cells $)$ of diluted suspensions of S. aureus. Groups of seven mice were used for each strain tested. Mice death was monitored every day for 7 weeks.

Animal studies were performed in accordance with the European Community guiding in the care and use of animals (Directive 2010/63/EU). Protocols were approved by the ethics committee of the Public University of Navarra ("Comité de Ética, Experimentación Animal y Bioseguridad" of the Universidad Pública de Navarra). Work was carried out in the animal facility of the Instituto de Agrobiotecnología, Universidad Pública de Navarra. Animals were housed under controlled environmental conditions with food and water ad libitum. Mice were euthanized by $\mathrm{CO}_{2}$ inhalation followed by cervical dislocation and all efforts were made to minimize suffering.

In these animal studies randomization methods were not used, and authors were not blinded to the group allocation during the experiment and when assessing the outcome.

Protein A expression analysis. To analyze protein A expression in CDM and RPMI media, overnight cultures were adjusted to an $\mathrm{OD}_{600 \mathrm{~nm}}$ of 0.1 , diluted $1 / 40$ in $\mathrm{CDM}$ or RPMI media, respectively, and incubated at $37^{\circ} \mathrm{C}$ without shaking for 24 $\mathrm{h}$ in sterile 24-well polystyrene microtiter plates (Sarstedt). A volume of $12 \mathrm{ml}$ of these bacterial cultures were centrifuged and pellets were washed with PBS and resuspended in $120 \mu \mathrm{l}$ PBS. Then, $1 \mu \mathrm{l}$ of lysostaphin $10 \mathrm{mg} \mathrm{ml}^{-1}$ (Sigma) and $1 \mu \mathrm{l}$ of TURBO DNase 2 units per ml (Ambion) were added. After $5 \mathrm{~h}$ of incubation at $37^{\circ} \mathrm{C}$, cell lysates were centrifuged and supernatants were collected. Protein concentration was determined with the Bio-Rad protein assay (Bio-Rad). Samples were adjusted to 3-5 $\mu \mathrm{g}$ of total protein and one volume of Laemmli buffer was added. Protein extracts were denatured by boiling at $100{ }^{\circ} \mathrm{C}$ for $5 \mathrm{~min}$. Proteins were separated on $12 \%$ SDS-polyacrylamide gels and stained with $0.25 \%$ Coomassie brilliant blue R250 (Sigma) or Criterion TGX Stain-Free Precast Gels (Bio-Rad Cat\#5678044). For western blotting, proteins were transferred onto Hybond-ECL nitrocellulose membranes (Amersham Biosciences) by semi-dry electroblotting. Membranes were blocked overnight with 5\% skimmed milk in PBS with $0.1 \%$ Tween 20 , and incubated with goat anti-mouse secondary antibodies labeled with horseradish peroxidase (Sigma) diluted 1:2500 for $1 \mathrm{~h}$ at room temperature. Protein A was detected with the SuperSignal West Pico Chemiluminescent Substrate (Thermo Fisher Scientific). To analyze protein A expression in TSB medium, overnight cultures were grown in TSB medium at $37^{\circ} \mathrm{C}$ with shaking at $200 \mathrm{rpm}$. A volume of $5 \mathrm{ml}$ of bacterial cultures were centrifuged and pellets were washed with PBS and resuspended in $120 \mu \mathrm{l}$ PBS. Then, cells were lysed and protein extracts were prepared as described above. Protein A expression was detected as described above. Densitometry quantification of the protein A western blotting bands from MW2 and $\Delta a r l S$ strains growing in the presence or absence of colistin was determined using ImageJ program. Ratios of the protein A band intensity from the strains growing in the presence or absence of colistin were calculated dividing the protein A band intensity from the strain growing in the presence of colistin by the protein A band intensity from the strain growing in absence of colistin. The average and SD of three independent assays were recorded.

RNA extractions. To analyze the spa RNA levels in CDM medium, overnight cultures were adjusted to an $\mathrm{OD}_{600 \mathrm{~nm}}$ of 0.1 , diluted $1 / 40$ in $\mathrm{CDM}$ medium, and incubated at $37^{\circ} \mathrm{C}$ without shaking for $24 \mathrm{~h}$ in sterile 24 -well polystyrene microtiter plates (Sarstedt). A volume of $12 \mathrm{ml}$ of these bacterial cultures were centrifuged and pellets were frozen in liquid nitrogen and stored at $-80^{\circ} \mathrm{C}$ until needed.

To analyze the spa RNA levels in TSB medium, overnight cultures were grown in TSB medium at $37^{\circ} \mathrm{C}$ with shaking at $200 \mathrm{rpm}$. A volume of $5 \mathrm{ml}$ of bacterial cultures were centrifuged, the pellets were frozen in liquid nitrogen, and stored at $-80^{\circ} \mathrm{C}$ until needed. Total RNA from bacterial pellets was extracted using the TRIzol reagent method as described ${ }^{62}$. RNA concentrations were quantified and RNAs were stored at $-80^{\circ} \mathrm{C}$ until needed.

Real-time quantitative PCR experiments. The spa RNA was quantified by realtime quantitative PCR (RT-qPCR) using AriaMx Real-Time PCR System and GoTaq 1-Step RT-qPCR System kit (Promega Ref: A6020). spa RNA was amplified with spa-FW and spa-RV primers, and gyrb RNA was used as endogenous controls using primers $g y r$-FW and $g y r-\mathrm{RV}^{63}$ (Supplementary Table 5). The specificity of RT-qPCR products was monitorized through the analysis of melting curves and electrophoresis. Only samples with no gyrB amplification of the minus reverse transcriptase aliquot were considered in the study. The amount of spa RNA was expressed as $2^{-\Delta \Delta C t}$, where $\Delta \mathrm{Ct}$ represents the difference in threshold cycle between the target and control (gyrase) genes and $\Delta \Delta \mathrm{Ct}$ represents the difference in $\Delta \mathrm{Ct}$ between the studying strain and the wild-type strain.

Hemolysis assay. To analyze hemolysins production, overnight cultures grown in TSB medium at $37^{\circ} \mathrm{C}$ were diluted in TSB to an $\mathrm{OD}_{600 \mathrm{~nm}}$ of 1 , and $5 \mu \mathrm{l}$ of diluted cultures were spotted onto $5 \%$ sheep blood Columbia agar plates (bioMérieux) and 5\% rabbit blood TSA plates (TSA from Pronadisa supplemented with defibrinated rabbit blood). Plates were incubated at $37^{\circ} \mathrm{C}$ for $24 \mathrm{~h}$. Hemolysins production was observed by the appearance of a clear halo.

Quantification of nitrite production. Bacterial strains were cultured in TSB media with $20 \mathrm{mM}$ potassium nitrate $\left(\mathrm{KNO}_{3}\right)$ under decreased oxygen tension condition that were created by growing the bacteria in $15 \mathrm{ml}$ Falcon tubes completely filled with medium ${ }^{64}$. Strains were incubated overnight at $37^{\circ} \mathrm{C}$ with shaking $(200 \mathrm{rpm}$ ) and the presence of nitrites in the media was determined colorimetrically by the Griess Reagent System (Promega) according to the manufacturer's instructions. A purple/magenta color indicates the presence of nitrites in the media. Representative pictures were taken.

Construction of plasmids expressing HK and/or RR genes. To construct the plasmids expressing the RRs, we amplified them by PCR using the corresponding forward (Fw) and reverse (Rv) primers (Supplementary Table 5) and the MW2 chromosomal DNA as template. Forward primers carried a SalI-XhoI tail and reverse primers carried a BamHI-XmaI-KpnI tail. PCR products were amplified with Phusion ${ }^{\otimes}$ High-Fidelity DNA Polymerase (Thermo Fisher Scientific), purified, and cloned in $\mathrm{pCR}^{\circledR}$-Blunt II TOPO vector (Invitrogen). Fragments were then ligated using SalI and KpnI enzymes in plasmid pCN51 ( $\mathrm{P}_{\text {cad }}$ promoter $)^{65}$ and electroporated into E. coli XL1-Blue (Stratagene) to generate the pCN51::RRn plasmid collection (Supplementary Table 4).

Similarly, HKs were amplified by PCR with the corresponding Fw and Rv primers (Supplementary Table 5) and the MW2 chromosomal DNA as template. Forward primers carried a BamHI tail and reverse primers carried a XmaI-AscI tail PCR products were amplified with Phusion ${ }^{\circledR}$ High-Fidelity DNA Polymerase (Thermo Fisher Scientific), purified, and cloned in $\mathrm{pCR}^{\circledast}$-Blunt II TOPO vector (Invitrogen).

For the construction of plasmids expressing a combination of HKs and RRs, TOPO HKn plasmids were digested with BamHI and XmaI enzymes, and the Bam HI/XmaI module, containing the HK, was transferred to the corresponding plasmid pCN51::RRn and electroporated into E. coli XL1-Blue to generate the pCN51::RRn-HKn plasmid collection (Supplementary Table 4).

The plasmid expressing ArlR with D52A amino-acid substitution was constructed by amplifying separately two DNA fragments by PCR using ArlR-Fw/ $\operatorname{ArlR}(D 52 A)-R v$ and ArlR(D52A)-Fw/ArlR-Rv primer pairs. These two PCR products were used as templates in an overlapping PCR, using ArlR-Fw/ArlR-Rv primers to amplify the mutant allele. The arlR(D52A) allele was cloned in pCN51 plasmid as we have described above for the construction of plasmids expressing RRs.

The plasmid expressing ArlS with H242A amino-acid substitution was constructed by amplifying separately two DNA fragments by PCR using ArlS-Fw/ ArlS(H242A)-Rv and ArlS(H242A)-Fw/ArlS-Rv primer pairs. These two PCR products were used as templates in an overlapping PCR, using ArlS-Fw/ArlS-Rv primers to amplify the mutant allele. The ArlS(H242A) allele was cloned in pCN51::arlR plasmid as we have described above for the construction of plasmids expressing HKs and RRs.

Plasmids were purified from E. coli XL1-Blue and transformed into S. aureus strains by electroporation.

The pCN51 inducible plasmid shows a basal expression in the absence of cadmium. All the experiments performed in this study that involve the pCN51 plasmid were carried out without cadmium supplementation.

Desiccation experiment. The desiccation experiment was adapted from an already described protocol ${ }^{66}$. Briefly, $1 \mathrm{ml}$ from overnight cultures grown in TSB medium at $37^{\circ} \mathrm{C}$ was harvested by centrifugation, washed with PBS three times, and finally resuspended in $1 \mathrm{ml}$ of PBS. The number of viable cells in this initial bacterial suspension was tested immediately (initial numbers), performing serial dilutions and plating dilutions in triplicate, and $100 \mu \mathrm{l}$ of this initial bacterial suspension were air-dried in 24-well tissue culture plates. Plates were stored at room temperature for 7 days. Dried bacteria were then rehydrated with $500 \mu$ l TSB and the number of viable cells remaining in each sample was determined by performing serial dilutions of each sample and plating the dilutions in triplicate. The average and SD of three independent assays were recorded.

Statistical analysis. All statistical analyses were performed in GraphPad Prism 5.01. Data corresponding to the kidney colonization assay were compared using a 
two-tailed nonparametric Mann-Whitney test. Data corresponding to the mice survival assay were compared using a log-rank (Mantel-Cox) test. Ratios of protein A quantification by western blot and data from qRT-PCR analysis were statistically analyzed using a two-tailed one-sample $t$-test inferred from a hypothetical value of 1. Finally, data corresponding to desiccation resistance were compared using a twotailed nonparametric Mann-Whitney test.

Data availability. Genomic sequence data for strains MW2, MW2 $\Delta \mathrm{XV}, \mathrm{RN} 1$, and RN1 $\Delta$ XV have been deposited in the National Center for Biotechnology Information Sequence Read Archive under accession code SRP127351. Differences in the metabolome of $S$. aureus isolate MW2 and $\triangle \mathrm{XV}$ mutant derivative have been deposited at figshare (https://doi.org/10.6084/m9.figshare.5733495.v1). Other relevant data are available in this article and its Supplementary Information files, or from the corresponding author upon request.

Received: 18 August 2016 Accepted: 10 January 2018

Published online: 06 February 2018

\section{References}

1. Stock, A. M., Robinson, V. L. \& Goudreau, P. N. Two-component signal transduction. Annu. Rev. Biochem. 69, 183-215 (2000).

2. Gao, R. \& Stock, A. M. Biological insights from structures of two-component proteins. Annu. Rev. Microbiol. 63, 133-154 (2009).

3. Capra, E. J. \& Laub, M. T. Evolution of two-component signal transduction systems. Annu. Rev. Microbiol. 66, 325-347 (2012).

4. Gotoh, Y. et al. Two-component signal transduction as potential drug targets in pathogenic bacteria. Curr. Opin. Microbiol. 13, 232-239 (2010).

5. Galperin, M. Y. A census of membrane-bound and intracellular signal transduction proteins in bacteria: bacterial IQ, extroverts and introverts. BMC Microbiol. 5, 35 (2005).

6. Ulrich, L. E. \& Zhulin, I. B. The MiST2 database: a comprehensive genomics resource on microbial signal transduction. Nucleic Acids Res. 38, D401-D407 (2010).

7. Alm, E., Huang, K. \& Arkin, A. The evolution of two-component systems in bacteria reveals different strategies for niche adaptation. PLoS Comput. Biol. 2 , e143-e143 (2006).

8. Skerker, J. M. et al. Rewiring the specificity of two-component signal transduction systems. Cell 133, 1043-1054 (2008).

9. Wertheim, H. et al. The role of nasal carriage in Staphylococcus aureus infections. Lancet Infect. Dis. 5, 751-762 (2005).

10. Lowy, F. D. Staphylococcus aureus infections-reply. N. Engl. J. Med. 339, 2026-2027 (1998).

11. Novick, R. P. Autoinduction and signal transduction in the regulation of staphylococcal virulence. Mol. Microbiol. 48, 1429-1449 (2003).

12. Cheung, A. L., Bayer, A. S., Zhang, G., Gresham, H. \& Xiong, Y.-Q. Regulation of virulence determinants in vitro and in vivo in Staphylococcus aureus. FEMS Immunol. Med. Microbiol. 40, 1-9 (2004).

13. Haag, A. F. \& Bagnoli, F. The role of two-component signal transduction systems in Staphylococcus aureus virulence regulation. Curr. Top. Microbiol. Immunol. 409, 145-198 (2016).

14. Galperin, M. Y., Higdon, R. \& Kolker, E. Interplay of heritage and habitat in the distribution of bacterial signal transduction systems. Mol. BioSyst. 6, 721-728 (2010).

15. Barakat, M. et al. P2CS: a two-component system resource for prokaryotic signal transduction research. BMC Genomics 10, 315 (2009).

16. Martin, P. K., Li, T., Sun, D., Biek, D. P. \& Schmid, M. B. Role in cell permeability of an essential two-component system in Staphylococcus aureus. J. Bacteriol. 181, 3666-3673 (1999).

17. Dubrac, S. \& Msadek, T. Identification of genes controlled by the essential YycG/YycF two-component system of Staphylococcus aureus. J. Bacteriol. 186, 1175-1181 (2004).

18. Toledo-Arana, A. et al. Staphylococcus aureus develops an alternative, icaindependent biofilm in the absence of the arlRS two-component system. J. Bacteriol. 187, 5318-5329 (2005)

19. Fournier, B., Klier, A. \& Rapoport, G. The two-component system ArlS-ArlR is a regulator of virulence gene expression in Staphylococcus aureus. Mol. Microbiol. 41, 247-261 (2001).

20. Novick, R. P. \& Geisinger, E. Quorum sensing in staphylococci. Annu. Rev. Genet. 42, 541-564 (2008).

21. Kinkel, T. L., Roux, C. M., Dunman, P. M. \& Fang, F. C. The Staphylococcus aureus SrrAB two-component system promotes resistance to nitrosative stress and hypoxia. MBio 4, e00696-13 (2013).

22. Wardenburg, J. B., Bae, T., Otto, M., DeLeo, F. R. \& Schneewind, O. Poring over pores: alpha-hemolysin and Panton-Valentine leukocidin in Staphylococcus aureus pneumonia. Nat. Med. 13, 1405-1406 (2007).
23. Skerker, J. M., Prasol, M. S., Perchuk, B. S., Biondi, E. G. \& Laub, M. T. Twocomponent signal transduction pathways regulating growth and cell cycle progression in a bacterium: a system-level analysis. PLoS Biol. 3, e334 (2005).

24. Laub, M. T., Biondi, E. G. \& Skerker, J. M. Phosphotransfer profiling: systematic mapping of two-component signal transduction pathways and phosphorelays. Methods Enzymol. 423, 531-548 (2007).

25. Yamamoto, K. et al. Functional characterization in vitro of all two-component signal transduction systems from Escherichia coli. J. Biol. Chem. 280, 1448-1456 (2005).

26. Procaccini, A., Lunt, B., Szurmant, H., Hwa, T. \& Weigt, M. Dissecting the specificity of protein-protein interaction in bacterial two-component signaling: orphans and crosstalks. PLoS ONE 6, e19729-e19729 (2011)

27. Capra, E. J., Perchuk, B. S., Skerker, J. M. \& Laub, M. T. Adaptive mutations that prevent crosstalk enable the expansion of paralogous signaling protein families. Cell 150, 222-232 (2012).

28. Biondi, E. G. et al. Regulation of the bacterial cell cycle by an integrated genetic circuit. Nature 444, 899-904 (2006).

29. Podgornaia, A. I., Casino, P., Marina, A. \& Laub, M. T. Structural basis of a rationally rewired protein-protein interface critical to bacterial signaling. Structure 21, 1636-1647 (2013).

30. Skarphol, K., Waukau, J. \& Forst, S. A. Role of His243 in the phosphatase activity of EnvZ in Escherichia coli. J. Bacteriol. 179, 1413-1416 (1997).

31. Hsing, W. H. \& Silhavy, T. J. Function of conserved histidine-243 in phosphatase activity of EnvZ, the sensor for porin osmoregulation in Escherichia coli. J. Bacteriol. 179, 3729-3735 (1997).

32. Falord, M., Mäder, U., Hiron, A., Débarbouillé, M. \& Msadek, T. Investigation of the Staphylococcus aureus GraSR regulon reveals novel links to virulence, stress response and cell wall signal transduction pathways. PLoS ONE 6, e21323 (2011)

33. Siryaporn, A. \& Goulian, M. Cross-talk suppression between the CpxA-CpxR and EnvZ-OmpR two-component systems in E. coli. Mol. Microbiol. 70, 494-506 (2008).

34. Silva, J. C., Haldimann, A., Prahalad, M. K., Walsh, C. T. \& Wanner, B. L. In vivo characterization of the type $\mathrm{A}$ and $\mathrm{B}$ vancomycin-resistant enterococci (VRE) VanRS two-component systems in Escherichia coli: a nonpathogenic model for studying the VRE signal transduction pathways. Proc. Natl Acad. Sci. USA 95, 11951-11956 (1998).

35. Groban, E. S., Clarke, E. J., Salis, H. M., Miller, S. M. \& Voigt, C. A. Kinetic buffering of cross talk between bacterial two-component sensors. J. Mol. Biol. 390, 380-393 (2009).

36. Siryaporn, A., Perchuk, B. S., Laub, M. T. \& Goulian, M. Evolving a robust signal transduction pathway from weak cross-talk. Mol. Syst. Biol. 6, 452 (2010).

37. Casino, P., Rubio, V. \& Marina, A. Structural insight into partner specificity and phosphoryl transfer in two-component signal transduction. Cell 139, 325-336 (2009).

38. Fournier, B. \& Hooper, D. C. A new two-component regulatory system involved in adhesion, autolysis, and extracellular proteolytic activity of Staphylococcus aureus. J. Bacteriol. 182, 3955-3964 (2000).

39. Watson, S. P., Clements, M. O. \& Foster, S. J. Characterization of the starvation-survival response of Staphylococcus aureus. J. Bacteriol. 180 , 1750-1758 (1998).

40. Delaune, A. et al. The WalKR system controls major staphylococcal virulence genes and is involved in triggering the host inflammatory response. Infect. Immun. 80, 3438-3453 (2012).

41. Dubrac, S., Boneca, I. G., Poupel, O. \& Msadek, T. New insights into the WalK/WalR (YycG/YycF) essential signal transduction pathway reveal a major role in controlling cell wall metabolism and biofilm formation in Staphylococcus aureus. J. Bacteriol. 189, 8257-8269 (2007).

42. Tu Quoc, P. H. et al. Isolation and characterization of biofilm formationdefective mutants of Staphylococcus aureus. Infect. Immun. 75, 1079-1088 (2007).

43. Holland, L. M. et al. A staphylococcal GGDEF domain protein regulates biofilm formation independently of cyclic dimeric GMP. J. Bacteriol. 190, 5178-5189 (2008).

44. Corrigan, R. M., Abbott, J. C., Burhenne, H., Kaever, V. \& Grundling, A. C-diamp is a new second messenger in Staphylococcus aureus with a role in controlling cell size and envelope stress. PLoS Pathog. 7, e1002217 (2011).

45. Foster, T. J., Geoghegan, J. A., Ganesh, V. K. \& Höök, M. Adhesion, invasion and evasion: the many functions of the surface proteins of Staphylococcus aureus. Nat. Rev. Microbiol. 12, 49-62 (2014).

46. Wallmark, G., Arremark, I. \& Telander, B. Staphylococcus saprophyticus: a frequent cause of acute urinary tract infection among female outpatients. $J$. Infect. Dis. 138, 791-797 (1978).

47. David, M. Z. \& Daum, R. S. Community-associated methicillin-resistant Staphylococcus aureus: epidemiology and clinical consequences of an emerging epidemic. Clin. Microbiol. Rev. 23, 616-687 (2010). 
48. Cucarella, C. et al. Bap, a Staphylococcus aureus surface protein involved in biofilm formation. J. Bacteriol. 183, 2888-2896 (2001).

49. Schenk, S. \& Laddaga, R. Improved method for electroporation of Staphylococcus aureus. FEMS Microbiol. Lett. 73, 133-138 (1992).

50. Novick, R. P. Genetic systems in staphylococci. Methods Enzymol. 204, 587-636 (1991).

51. Ruiz de los Mozos, I. et al. Base pairing interaction between $5^{\prime}$ - and $3^{\prime}$-UTRs controls icaR mRNA translation in Staphylococcus aureus. PLoS Genet. 9, e1004001-e1004001 (2013).

52. Arnaud, M., Chastanet, A. \& Débarbouillé, M. New vector for efficient allelic replacement in naturally nontransformable, low-GC-content, Gram-positive bacteria. Appl. Environ. Microbiol. 70, 6887-6891 (2004).

53. Valle, J. et al. SarA and not sigmaB is essential for biofilm development by Staphylococcus aureus. Mol. Microbiol. 48, 1075-1087 (2003).

54. Marmur, J. A procedure for the isolation of deoxyribonucleic acid from microorganisms. Methods Enzymol. 6, 726-738 (1963).

55. Luo, R. et al. SOAPdenovo2: an empirically improved memory-efficient shortread de novo assembler. Gigascience 1, 18 (2012).

56. Rissman, A. I. et al. Reordering contigs of draft genomes using the Mauve Aligner. Bioinformatics 25, 2071-2073 (2009).

57. Barrick, J. E. et al. Genome evolution and adaptation in a long-term experiment with Escherichia coli. Nature 461, 1243-1247 (2009).

58. Tautenhahn, R., Böttcher, C. \& Neumann, S. Highly sensitive feature detection for high resolution LC/MS. BMC Bioinformatics 9, 504 (2008).

59. Scheltema, R. A., Jankevics, A., Jansen, R. C., Swertz, M. A. \& Breitling, R. PeakML/mzMatch: a file format, Java library, R library, and tool-chain for mass spectrometry data analysis. Anal. Chem. 83, 2786-2793 (2011).

60. Creek, D. J., Jankevics, A., Burgess, K. E. V., Breitling, R. \& Barrett, M. P. IDEOM: an Excel interface for analysis of LC-MS-based metabolomics data. Bioinformatics 28, 1048-1049 (2012).

61. Mccallum, N., Meier, P. S., Heusser, R. \& Berger-Bachi, B. Mutational analyses of open reading frames within the vraSR operon and their roles in the cell wall stress response of Staphylococcus aureus. Antimicrob. Agents Chemother. 55, 1391-1402 (2011).

62. Lasa, I. et al. Genome-wide antisense transcription drives mRNA processing in bacteria. Proc. Natl Acad. Sci. USA 108, 20172-20177 (2011).

63. Wolz, C., Goerke, C., Landmann, R., Zimmerli, W. \& Flückiger, U. Transcription of clumping factor A in attached and unattached Staphylococcus aureus in vitro and during device-related infection. Infect. Immun. 70, 2758-2762 (2002).

64. Schlag, S. et al. Characterization of the oxygen-responsive NreABC regulon of Staphylococcus aureus. J. Bacteriol. 190, 7847-7858 (2008).

65. Charpentier, E. et al. Novel cassette-based shuttle vector system for Grampositive bacteria. Appl. Environ. Microbiol. 70, 6076-6085 (2004).

66. White, A., Gibson, D., Kay, W. \& Surette, M. Thin aggregative fimbriae and cellulose enhance long-term survival and persistence of Salmonella. J. Bacteriol. 188, 3219-3227 (2006).

\section{Acknowledgements}

We thank Alberto Marina for the in vitro phosphorylation assays; Victor Segura for data processing in the sequencing of the strains; Tarek Msadek, Tino Krell, William Kelley, Michael Galperin, Jean Marc Ghigo, Victor de Lorenzo, and Josep Casadesús for critical reading of the paper; and Ambrose L. Cheung for providing the COL strain and its $\Delta$ arl mutant. This work was supported by the Spanish Ministry of Economy and Competitiveness grants BIO2011-30503-C02-02, BIO2014-53530-R, SAF2014-56716-REDT , and RTC-2015-3184-1. J.V. was supported by Ramon y Cajal (RYC-2009-03948) contract from the Spanish Ministry of Economy and Competitiveness.

\section{Author contributions}

M.V., B.G., J.V., C.S., J.R.P., A.T.-A. and I.L. designed research; M.V., B.G., B.R. and M. M. performed research; M.V., B.G., I.R.M., J.R.P., A.T.-A. and I.L. analyzed data; and M. V., B.G. and I.L. wrote the paper.

\section{Additional information}

Supplementary Information accompanies this paper at https://doi.org/10.1038/s41467018-02949-y.

Competing interests: The authors declare no competing financial interests.

Reprints and permission information is available online at http://npg.nature.com/ reprintsandpermissions/

Publisher's note: Springer Nature remains neutral with regard to jurisdictional claims in published maps and institutional affiliations.

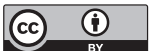

Open Access This article is licensed under a Creative Commons Attribution 4.0 International License, which permits use, sharing, adaptation, distribution and reproduction in any medium or format, as long as you give appropriate credit to the original author(s) and the source, provide a link to the Creative Commons license, and indicate if changes were made. The images or other third party material in this article are included in the article's Creative Commons license, unless indicated otherwise in a credit line to the material. If material is not included in the article's Creative Commons license and your intended use is not permitted by statutory regulation or exceeds the permitted use, you will need to obtain permission directly from the copyright holder. To view a copy of this license, visit http://creativecommons.org/ licenses/by/4.0/.

(C) The Author(s) 2018 